\title{
Correspondence
}

\section{Anesthesia research productivity in Canadian universities: a full picture?} (I)

To the Editor:

We are grateful that Tsui et al. ${ }^{1}$ have presented serious concerns about the status of anesthesia research in Canada in the March 2006 issue of the Journal. We believe that a resident's decision to pursue research fellowship training and a research career depends on many factors, including the desire for an academic career (which probably depends on exposure and role modelling during residency), lifestyle, current debt, future income expectations, institutional culture, and research infrastructure and support. With the current anesthesia shortage, it is difficult to convince an outstanding resident to take on additional years of research training if the institution or department derogates the value of research and researchers. This is particularly problematic in institutions where a practice plan or an alternative funding program is not available to protect academic time.

Although Tsui et al. provide interesting information, we are concerned about the inaccurate portrayal of "publication rates" for various universities, which appears to paint a biased picture of research prowess of the bigger institutions. Tsui and colleagues suggest that limitations related to collaborative publication across departments "would be common to all Canadian universities in our search", but we believe that is incorrect. In their report, Dalhousie was credited with only 13 papers in five years. However, a quick search in Medline revealed that just three of the several researchers in our institution had a total of 53 publications during that period of time. So the reported number grossly underestimates the productivity of our institution, hardly a "slight under representation". Perhaps, this is, in part, due to the search criteria used in their study (i.e., searching for "anesthesia" or its variants in the "address" field on Medline). It may be more common for researchers in small departments to collaborate with colleagues in other disciplines simply because there is less range of expertise within their own department, or to supervise research students from other disciplines. For example, many of our faculty members have cross appointments in other departments (e.g., psychology, pharmacology, surgery, internal medicine, critical care, and pain research), so corresponding addresses may not have included the term "anesthesia". If Tsui et al. have reported an equivalent four- or fivefold underestimate of publication numbers for all the universities in their study, then there is little support for their conclusion that anesthesia research has been static over the time period, nor for their interpretation of publication types. If their inaccuracy is disproportionately focused on smaller departments, as we believe, then there is a clearly biased portrayal of research productivity that may be directly harmful, particularly to faculty recruitment efforts. In any case, reporting a "lack" of research productivity using an inaccurate measure and a narrow search strategy is potentially damaging to our specialty.

Notwithstanding these issues, we would echo the investigators' concerns about the future of Canadian anesthesia research. A strategic plan to address funding issues, research infrastructure, and institutional culture is certainly needed.

\section{G. Allen Finley MD FRCPC FAAP}

Orlando Hung MD FRCPC

Thomas J. Coonan MD FRCPC

Richard Hall MD FRCPC

Dalhousie University, Halifax, Canada

E-mail: allen.finley@dal.ca

Accepted for publication May 2, 2006.

\section{Reference}

I Tsui BC, Li LX, Ma V, Wagner AM, Finucane BT. Declining randomized clinical trials from Canadian anesthesia departments? Can J Anesth 2006; 53: 22635 .

\section{Anesthesia research productivity in Canadian universities: a full picture?} (II)

To the Editor:

Tsui et al. ${ }^{1}$ reported on the number of published articles from the Canadian departments of anesthe- 
siology for the period 2000-2004, and mention the increasing productivity of Université de Montréal. It is encouraging to have our efforts recognized but we believe that Tsui et al. underestimated our research achievements.

According to its annual reports for 2000-2004, our department had 229 publications, with a high in 2001 (55) and a low in 2003 (35). No apparent upward trend is seen, contrary to Tsui et al.'s observation. However, the methodology used for the annual report is different from Tsui et al.'s. We enter all articles authored by at least one member of our department, including those written in collaboration with another department or university. However, the large discrepancy between our annual report and Tsui et al.'s finding prompted us to investigate the matter in more detail.

We searched the MEDLINE database using the PubMed search engine, as done by Tsui et al., ${ }^{1}$ using the same keywords, except that location was limited to Montreal. For the years 2000-2004, the search returned 171 entries, of which 82 (all included in our annual reports) were identified as coming from Université de Montréal. Tsui et al. reported 86, with an identical breakdown according to year of publication except for 2002 and 2004. The discrepancies are explained by Letters to the Editor (27); work attributed to another university (e.g., during a fellowship) or performed in collaboration with another department or university (86); missing address of authors of editorials or commentaries (12); identification of the department in French (13); and failure of the search engine to retrieve relevant articles (9). Most of these factors probably played a role for other university anesthesia departments as well, suggesting that publications from Canadian anesthesiologists as a whole might have been underestimated by Tsui et al.

Tsui et al. also mentioned the importance of research during residency training and the key role of mentorship. At Université de Montréal, a threemonth period without any clinical duties is set aside to complete a research project. Between 2000 and 2004, residents appeared as first author on 32 articles. Of the 38 residents who completed a research rotation between 1997 and 2002, 25 (66\%) published a peerreviewed article and $35(92 \%)$ presented their work as an abstract and/or full length paper. Thus, residents contribute significantly to the research productivity of our department and, more importantly, to the growth of knowledge within our specialty.

François Donati PhD MD FRCPC Jean-François Hardy MD FRCPC
Université de Montréal, Montréal, Canada

E-mail: francois.donati@umontreal.ca Accepted for publication May 5, 2006.

\section{Reference}

Tsui BC, Li LX, Ma V, Wagner AM, Finucane BT. Declining randomized clinical trials from Canadian anesthesia departments? Can J Anesth 2006; 53 : 226-35.

\section{Anesthesia research productivity in Canadian universities: a full picture? (III)}

To the Editor:

I appreciate the recent article by Tsui et al. ${ }^{1}$ which highlights important threats and recent challenges facing academic anesthesiology. In their observational study, the authors rated the number of publications amongst Canadian university departments. They concluded that the numbers of randomized clinical trials conducted in Canadian departments are decreasing, although the overall numbers of anesthesia publications have not declined significantly. The authors highlight the importance of encouraging anesthesia residents to undertake a dedicated research rotation, the importance of creating an academic environment with protected non-clinical time, and the role of mentorship

I echo their serious concerns regarding the future of our specialty. Without advancing the knowledge base of anesthesia through research, our specialty risks gradual deterioration into a clinical service and technical department, in comparison with our academic peers. However, I have major reservations in the methodology and benchmark metrics on research productivity as presented by Tsui et al. ${ }^{1}$

Firstly, the study grossly underestimated the number of research publications from the Department of Anesthesia at University of Western Ontario (UWO) for the period 2000-2004. In comparing our annual reports and repeating the MEDLINE search, the UWO Department published 97 peer-reviewed publications instead of 37 as reported by Tsui and colleagues during the five-year sampling frame. Using the definition of study design in Table I of Tsui et al., the UWO Department actually published 13 laboratory investigations, four basic sciences studies, six case reports, four case series, four clinical trials, four cohort studies, five editorials, four invited commentaries, one meta-analysis, one non-clinical study, eight randomized controlled trials (RCT), four multicentre RCTs, 